\title{
LIFT ZONOID AND BARYCENTRIC REPRESENTATION ON A BANACH SPACE WITH A CYLINDER MEASURE
}

\author{
ALEXEI M. KULIK AND TARAS D. TYMOSHKEVYCH
}

\begin{abstract}
We show that the lift zonoid concept for a probability measure on $\mathbb{R}^{d}$, introduced in (Koshevoy and Mosler, 1997), leads naturally to a one-to one representation of any interior point of the convex hull of the support of a continuous measure as the barycenter w.r.t. to this measure of either of a half-space, or the whole space. We prove the infinite-dimensional generalization of this representation, which is based on the extension of the lift-zonoid concept for a cylindrical probability measure.
\end{abstract}

\section{INTRODUCTION}

For a probability measure $\mu$ with a finite first moment, defined on the Borel $\sigma$-algebra in $\mathbb{R}^{d}$, its zonoid $Z(\mu)$ is defined as the set of all the points in $\mathbb{R}^{d}$ of the form

$$
\int_{\mathbb{R}^{d}} g(x) x \mu(d x)
$$

with arbitrary measurable $g: \mathbb{R}^{d} \rightarrow[0,1]$, see [5], Definition 2.1. The lift zonoid $\hat{Z}(\mu)$ is defined as the zonoid of the measure $\delta_{1} \times \mu$ in $\mathbb{R}^{d+1}$. Equivalently, the zonoid $Z(\mu)$ and the lift zonoid $\hat{Z}(\mu)$ are the sets of the points of the form

$$
E g(X) X \in \mathbb{R}^{d} \text { and }(E g(X), E g(X) X) \in \mathbb{R}^{d+1}
$$

respectively, where $X$ is a random vector with the distribution $\mu$. The zonoid $Z(\mu)$ (resp., the lift zonoid $\hat{Z}(\mu)$ ) is a convex compact set in $\mathbb{R}^{d}$ (resp., $\mathbb{R}^{d+1}$ ), symmetric w.r.t. the point $(1 / 2) E X$ (resp., the point $((1 / 2),(1 / 2) E X))$.

Zonoid and lift zonoid concepts appear to be useful in various aspects. The lift zonoid determines the underlying measure uniquely; see [5], 4]. This important observation leads to natural applications of the lift zonoid concept in multivariate statistics for measuring the variability of laws of random vectors, and for ordering these laws, see [5. Although the zonoid of a measure does not determine the measure uniquely, the concept of zonoid equivalence appears to be both naturally motivated by financial applications, and useful for proving extensions of the ergodic theorem for zonoid stationary and zonoid swap-invariant random sequences, see [6], 7]. Lift zonoids also lead to natural definitions of associated $\alpha$-trimming and data depth, see [4].

In this paper, we are concentrated on the application of the lift zonoid concept to a barycentric representation of the points of the initial space w.r.t. the given measure $\mu$. The possibility of such an application was mentioned in [4]; however, respective Theorem 3.1 in [4] is not the best possible one. Below we show that, for a continuous measure $\mu$ (see condition (8) below), every interior point of the closed convex support $\mathbf{S}(\mu)$ of the measure $\mu$ has a unique representation as a barycenter either of a half-space, or of the whole space. This barycentric representation has the same spirit with the classical Krein-Milman theorem, see e.g. 8], Chapter 3, but unlike the Krein-Milman theorem has an important uniqueness feature. In some particularly interesting cases, this makes it possible to introduce the barycentric coordinates on the initial space.

The lift zonoid concept can be extended naturally to the infinite-dimensional setting, and such an extension appears to be particularly useful. For instance, it was shown in 2 that the (analogue of) the lift zonoid induced by the Brownian motion can be used in the sensitivity

Key words and phrases. Zonoid, lift zonoid, cylinder measure, barycentric representation. 
analysis of a certain iterative portfolio selection problem. In this paper, we show that infinitedimensional lift zonoids can be used efficiently to obtain a barycentric representation on a Banach space. It is important observation that, in order to obtain the barycentric representation in its most closed and simple form, one needs to consider a cylinder probability measure $\mu$ on the initial space, rather than a usual probability measure. This leads naturally for the extension of the lift zonoid concept for cylinder probability measures.

The structure of the paper is the following. In Section 2, we introduce the notions of the zonoid, the lift zonoid, and the zonoid trimmed regions, induced by a cylinder probability measure on a Banach space. We give the basic properties of these objects, and formulate the main result of this paper about the barycentric representation w.r.t. to the given cylinder measure $\mu$. The proofs of these results are given in Section 3. In Section 4, we discuss the way how does the barycentric representation lead to three closely related types of barycentric coordinates on the initial space. The barycentric representation from Section 2 relies on the assumption that the zonoid span $\mathcal{L}(\mu)$ coincides with the initial space; see the definition and the discussion in Remark 3. For a $\sigma$-additive measure $\mu$ such an assumption typically fails; in Section 5 we formulate the version of the barycentric representation theorem for a space with a $\sigma$-additive measure $\mu$, which takes into account the natural topology of the zonoid span $\mathcal{L}(\mu)$. In Section 6 , we consider an illustrative example with $\mu$ being either a centered cylinder Gaussian measure with identity covariance operator, or a centered $\sigma$-additive Gaussian measure.

\section{MAin RESUlts}

2.1. Infinite-dimensional zonoid, lift zonoid, and zonoid trimmed regions, induced by a cylinder probability measure. Let $\mathbb{X}$ be a reflexive separable Banach space. Denote by $\mathcal{C}$ its cylinder algebra; that is, the family of subsets in $\mathbb{X}$ of the form

$$
\left\{x \in \mathbb{X}:\left(\left\langle x, x_{1}^{*}\right\rangle, \ldots\left\langle x, x_{m}^{*}\right\rangle\right) \in B\right\}, \quad m \geq 1, \quad x_{1}^{*}, \ldots, x_{m}^{*} \in \mathbb{X}^{*}, \quad B \in \mathcal{B}\left(\mathbb{R}^{m}\right),
$$

here and below $\mathbb{X}^{*}$ denotes the dual space to $\mathbb{X}$, and $\langle\cdot, \cdot\rangle$ denotes the duality between $\mathbb{X}$ and $\mathbb{X}^{*}$. Let $\mu$ be a cylinder probability measure on $\mathcal{C}$ of type 1 (see [10], Chapter IV, $\S 5$ ), that is, an additive function $\mu: \mathcal{C} \rightarrow[0,1]$ such that

(a) for every $m \geq 1, x_{1}^{*}, \ldots, x_{m}^{*} \in \mathbb{X}^{*}$ the restriction of $\mu$ to the $\sigma$-algebra

$$
\mathcal{C}_{x_{1}^{*}, \ldots, x_{m}^{*}}:=\left\{\left\{x:\left(\left\langle x, x_{1}^{*}\right\rangle, \ldots\left\langle x, x_{m}^{*}\right\rangle\right) \in B\right\}, B \in \mathcal{B}\left(\mathbb{R}^{m}\right)\right\}=\sigma\left(\left\langle\cdot, x_{1}^{*}\right\rangle, \ldots,\left\langle\cdot, x_{m}^{*}\right\rangle\right)
$$

is a probability measure;

(b) there exists $C_{\mu}>0$ such that

$$
\int_{\mathbb{X}}\left|\left\langle x, x^{*}\right\rangle\right| \mu(d x) \leq C_{\mu}\left\|x^{*}\right\|_{\mathbb{X}^{*}}, \quad x^{*} \in \mathbb{X}^{*}
$$

The cylinder measure $\mu$ can be naturally interpreted as the "law" of a generalized random element $X$ in $\mathbb{X}$ with finite 1-st order, which, by definition, is the linear bounded map

$$
X: \mathbb{X}^{*} \rightarrow L_{1}(\Omega, \mathcal{F}, P),
$$

where $(\Omega, \mathcal{F}, P)$ is some probability space; see [10], Chapter IV for details. The mean value $\int_{\mathbb{X}} x \mu(d x)$ or, equivalently, the expectation $E[X]$ is well defined in the following sense. It can be seen easily that the map

$$
T: \mathbb{X}^{*} \ni x^{*} \mapsto \int_{\mathbb{X}}\left\langle x, x^{*}\right\rangle \mu(d x)=E\left\langle X, x^{*}\right\rangle \in \mathbb{R}
$$

is linear and continuous. Because $\mathbb{X}$ is assumed to be reflexive, there exists $x_{\mu} \in \mathbb{X}$ such that $T\left(x^{*}\right)=\left\langle x_{\mu}, x^{*}\right\rangle$. By the definition,

$$
\int_{\mathbb{X}} x \mu(d x)=E[X]=x_{\mu}
$$

Note that, by the same argument, for any bounded random variable $\eta$ the expectation $E[\eta X] \in \mathbb{X}$ is well defined. 
Definition 1. The zonoid $Z(\mu)$ of the cylinder measure $\mu$ is the set of all points in $\mathbb{X}$ of the form $E[\eta X]$ where $X$ is a generalized random element in $\mathbb{X}$ with the distribution $\mu$, and $\eta: \Omega \rightarrow[0,1]$ is arbitrary $\sigma(X)$-measurable random variable.

The lift zonoid $\hat{Z}(\mu)$ is the set of all points in $\mathbb{R} \times \mathbb{X}$ of the form $(E \eta, E[\eta X])$ with is arbitrary $\sigma(X)$-measurable random variable $\eta: \Omega \rightarrow[0,1]$.

For $\alpha \in(0,1]$, the zonoid $\alpha$-trimmed region $D_{\alpha}(\mu)$ is the set of all the points in $\mathbb{X}$ of the form

$$
\frac{1}{\alpha} E[\eta X], \quad g \in F_{\alpha},
$$

where $F_{\alpha}$ denotes the family of $\sigma(X)$-measurable random variables $\eta: \Omega \rightarrow[0,1]$ such that $E \eta=\alpha$.

Remark 1 . If $X$ is a random vector in $\mathbb{R}^{d}$, then any $\sigma(X)$-measurable random variable $\eta$ have the form $\eta=g(X)$ with a Borel measurable $g: \mathbb{R}^{d} \rightarrow \mathbb{R}$. Therefore, the above definition is a straightforward generalization of the finite-dimensional definitions of zonoid and lift zonoid, mentioned in Introduction.

In the following theorem, the basic properties of zonoid, lift zonoid, and zonoid $\alpha$-trimmed regions are collected. Denote by $\mathcal{H}^{\mathbb{X}}$ the class of all sets of the form $\left\{x \in \mathbb{X}:\left\langle x, x^{*}\right\rangle \geq a\right\}$ with $x^{*} \in \mathbb{X}, a \in \mathbb{R}$; clearly, every set $H \in \mathcal{H}^{\mathbb{X}}$ is a cylinder set. Denote also

$$
\mathbf{S}(\mu)=\bigcap_{H \in \mathcal{H}^{\mathbb{X}}: \mu(H)=1} H
$$

note that, in the case of $\mathbb{X}=\mathbb{R}^{d}$ and a (usual) probability measure $\mu$, the set $\mathbf{S}(\mu)$ coincides with the closed convex hull of the topological support of $\mu$.

Theorem 1. Let $\mu$ be a cylinder probability measure of type 1, and $X$ be respective generalized random element.

Then the following properties hold true.

(a) $Z(\mu)$ and $\hat{Z}(\mu)$ are bounded closed convex sets in $\mathbb{X}$ and $\mathbb{R} \times \mathbb{X}$, respectively.

(b) $Z(\mu)$ and $\hat{Z}(\mu)$ are symmetric w.r.t. the points $(1 / 2) E[X]$ and $((1 / 2),(1 / 2) E[X])$, respectively.

(c) $\hat{Z}(\mu)$ determines $\mu$ uniquely.

(d) Every $D_{\alpha}(\mu), \alpha \in(0,1]$ is a bounded closed convex compact set in $\mathbb{R}^{d}$.

(e) $D_{\alpha}(\mu) \supset D_{\beta}(\mu)$ for every $\alpha<\beta$.

(f) If the measure $\mu$ is centered (that is, $E[X]=0$ ), then

$$
\alpha D_{\alpha}(\mu)=-(1-\alpha) D_{1-\alpha}(\mu), \quad \alpha \in(0,1] .
$$

(g) For any sequence $\alpha_{n} \rightarrow \alpha \in(0,1)$,

$$
D_{\alpha_{n}}(\mu) \rightarrow D_{\alpha}(\mu)
$$

in the Hausdorff distance. At the point $\alpha=1$ the family $D_{\alpha}(\mu), \alpha \in(0,1]$ is continuous in the following weaker sense:

$$
D_{1}(\mu)=\bigcap_{\alpha \in(0,1)} D_{\alpha}(\mu)
$$

(h) The closure of $D_{0}(\mu):=\bigcup_{\alpha \in(0,1]} D_{\alpha}(\mu)$ equals $\mathbf{S}(\mu)$.

Theorem 1 is proved in Section 3 below.

It was mentioned in 2] briefly that, for a (usual) probability measure $\mu$ its zonoid is necessarily a compact. More precisely, this property can be formulated as follows.

Proposition 1. Let $\mu$ a probability measure such that the respective family of random variables

$$
\left\{\left\langle X, x^{*}\right\rangle,\left\|x^{*}\right\|_{\mathbb{X}^{*}} \leq 1\right\}
$$

is uniformly integrable.

Then $Z(\mu), \hat{Z}(\mu)$, and $D_{\alpha}(\mu), \alpha \in(0,1]$ are compact sets in $\mathbb{X}$. 
Proposition 1 is proved in Section 3 .

Remark 2. It is sufficient for the uniform integrability of the family (44) that either $\mu$ has a strong moment of order 1 ; that is

$$
E\|X\|<\infty,
$$

or $\mu$ has a weak moment of some order $p>1$; that is,

$$
E\left|\left\langle X, x^{*}\right\rangle\right|^{p}<\infty, \quad x^{*} \in \mathbb{X}^{*}
$$

Under the assumption that $\mu$ has a weak moment of order $p>1$, the compactness property, equivalent to that of Proposition 1, was proved in [3].

In the finite-dimensional setting, an alternative definition of the zonoid is the expectation of the random segment $[0, X]$, see [5]. To complete the list of main properties of the zonoid of a cylinder probability measure, we give the extension of this definition for the infinite-dimensional setting.

Denote by $\mathbb{S}_{\mathbb{X}^{*}}$ the unit sphere in $\mathbb{X}^{*}$, and by $h(C, \cdot)$ the support function of a convex set $C \subset \mathbb{X}:$

$$
h\left(C, x^{*}\right)=\sup \left\{\left\langle x, x^{*}\right\rangle, x \in C\right\}, \quad x^{*} \in \mathbb{S}_{\mathbb{X}^{*}} .
$$

Consider the "random segment" $[0, X]$, which is generalized in the sense that $X$ is a generalized random element in $\mathbb{X}$. Note that for every $x^{*} \in \mathbb{S}_{\mathbb{X}}$ respective value of the support function

$$
h\left([0, X], x^{*}\right)=\left\langle X, x^{*}\right\rangle_{+}:=\left(\left\langle X, x^{*}\right\rangle \vee 0\right)
$$

is measurable w.r.t. $\mathcal{C}_{x^{*}}$, dominated by $\left|\left\langle X, x^{*}\right\rangle\right|$, and therefore is integrable. Define the expectation $E[0, X]$ of $[0, X]$ as the convex closed set in $\mathbb{X}$ such that

$$
h\left(E[0, X], x^{*}\right)=E h\left([0, X], x^{*}\right), \quad x^{*} \in \mathbb{S}_{\mathbb{X}^{*}} .
$$

Proposition 2. (1) The set $E([0, X])$ is well defined by (7), and $Z(\mu)=E([0, X])$.

(2) $\hat{Z}(\mu)=E[0,(1, X)]$.

The proof of Proposition 2 is close to that of Proposition 2.2 in [5], with a minor difference caused by the fact $Z(\mu)$ is not necessarily a compact now; see Section 3 below.

2.2. Barycentric representation. In this section, introduce the barycentric representation in $\mathbb{X}$ w.r.t. to the given cylinder measure $\mu$. We assume that $\mu$ is continuous in the sense that

$$
\mu\left(\left\{x:\left\langle x, x^{*}\right\rangle=a\right\}\right)=0, \quad x^{*} \in \mathbb{X} \backslash\{0\}, \quad a \in \mathbb{R} .
$$

For a cylinder set $A$ with $\mu(A)>0$, its barycenter w.r.t. $\mu$ is defined by the identity

$$
B_{A}(\mu)=\frac{\int_{A} x \mu(d x)}{\mu(A)}
$$

(another term for the barycenter $B_{A}(\mu)$ is A-centroid of $\mu$, see [4]).

Theorem 2. Let $\mu$ be a cylinder probability measure of type 1. Assume that

(i) $\mu$ satisfy (8);

(ii) the interior of $D_{0}(\mu)$ is non-empty.

Then the following holds true.

(1) The set $\mathbf{B}(\mu)=\left\{B_{A}(\mu), \mu(A)>0\right\}$ of all barycenters w.r.t. $\mu$ equals the interior of $\mathbf{S}(\mu)$.

(2) Any point $x \in \mathbf{B}(\mu)$ has a representation in the form

$$
x=B_{H}(\mu), \quad H \in \mathcal{H}^{\mathbb{X}}, \quad \mu(H)>0 .
$$

This representation is unique in the following sense: if $B_{H}(\mu)=B_{G}(\mu)$ for some $H, G \in$ $\mathcal{H}^{\mathbb{X}}$, then

$$
\mu(H \Delta G)=0 .
$$


Theorem 2 is proved in Section 3 .

Remark 3. Condition (ii) can be reformulated in the following, more geometrical, way. Without loss of generality, we can assume $\mu$ to be centered. Denote by $\mathcal{L}(\mu)$ the linear span of $D_{0}(\mu)$. By statements (e), (f) of Theorem 1 and by the definition of $Z(\mu)$, one has

$$
\mathcal{L}(\mu)=\bigcup_{r \in(0, \infty)} r D_{1 / 2}(\mu)=\bigcup_{r \in(0, \infty)} r Z(\mu)
$$

Then (ii) is equivalent to the following:

$$
\mathcal{L}(\mu)=\mathbb{X}
$$

By the identity (10), the linear space $\mathcal{L}(\mu)$ is naturally endowed by the norm $\|\cdot\|_{\mathcal{L}}$ equal to the Minkowskii functional of the zonoid $Z(\mu)$; note that $Z(\mu)$ is symmetric and convex because $\mu$ is centered. We call the normed space $\mathcal{L}(\mu)$ with the norm $\|\cdot\|_{\mathcal{L}}$ the zonoid span of the measure $\mu$, and remark that condition (ii), in fact, means that the initial space $\mathbb{X}$ coincides with the zonoid span of $\mu$.

Remark 4 . In the case $\mathbb{X}=\mathbb{R}^{d}$ condition (ii') (and therefore (ii)) holds trivially: if (ii') fails then the measure is concentrated on the hyper-plane $\mathcal{L}(\mu)$, which contradicts to (i).

However, in the infinite-dimensional setting condition (ii) is much more demanding. For instance, this condition fails for any $\sigma$-additive probability measure $\mu$ on, say, an infinitedimensional Hilbert space, such that the respective family (4) is uniformly integrable. Indeed, in that case $D_{0}(\mu)$ is a $\sigma$-compact set, and therefore by the Baire category theorem has an empty interior. In other words, in that case the geometry of the zonoid span $\mathcal{L}(\mu)$ necessarily differs from the geometry of $\mathbb{X}$.

\section{Proofs}

In this section we prove Theorem 1, Proposition 1, and Theorem 2, Without loss of generality we assume $\mu$ to be centered.

3.1. Properties of zonoid and lift zonoid. Here we prove statements (a) - (c) of Theorem 1. Proposition 1, and Proposition 2.

Statements (a), (b). Clearly, it is enough to prove (a) and (b) for the zonoid $Z(\mu)$, only. Convexity of $Z(\mu)$ follows by the definition: if $x_{1}=E\left[\eta_{1} X\right]$ and $x_{2}=E\left[\eta_{2} X\right]$ are some points from $Z(\mu)$, then for any $c \in[0,1]$ the point $c x_{1}+(1-c) x_{2}=E\left[\left(c_{1} \eta_{1}+(1-c) \eta_{2}\right) X\right]$ belongs to $Z(\mu)$, as well. Symmetricity also follows by the definition: for any $x=E[\eta X] \in Z(\mu)$ the symmetric point $(-x)$ can be represented as $(-x)=E[(1-\eta) X]$, and therefore belongs to $Z(\mu)$.

Because for any $x=E[\eta X] \in Z(\mu)$ one has by (3)

$$
\left|\left\langle x, x^{*}\right\rangle\right| \leq E|\eta|\left|\left\langle X, x^{*}\right\rangle\right| \leq E\left|\left\langle X, x^{*}\right\rangle\right| \leq C_{\mu}\|x\|_{\mathbb{X}^{*}}^{*}, \quad x^{*} \in \mathbb{X}^{*},
$$

the set $Z(\mu)$ lies in the ball $\left\{x:\|x\|_{\mathbb{X}} \leq C_{\mu}\right\}$ and hence is bounded.

Let $x_{n}=E\left[\eta_{n} X\right], n \geq 1$ be a sequence in $Z(\mu)$. Consider the variables $\eta_{n}, n \geq 1$ as elements of the unit ball in the space $L_{\infty}(\Omega, \sigma(X), P)=\left(L_{1}(\Omega, \sigma(X), P)\right)^{*}$. Then by the Banach-Alaoglu theorem (see [8], Chapter 3 ) there exists a *-weakly convergent subsequence $\left\{\eta_{n_{k}}\right\}$, denote by $\hat{\eta}$ respective limit. Then

$$
\left\langle x_{n_{k}}, x^{*}\right\rangle=E \eta_{n_{k}}\left\langle X, x^{*}\right\rangle \rightarrow E \hat{\eta}\left\langle X, x^{*}\right\rangle, \quad x^{*} \in \mathbb{X}^{*} .
$$

Therefore, if $x_{n} \rightarrow x$ in $\mathbb{X}$, then $x=E[\hat{\eta} X] \in Z(\mu)$; that is, $Z(\mu)$ is closed.

Proposition 1. In fact, we have just proved that any sequence $\left\{x_{n}\right\} \subset Z(\mu)$ has a subsequence $\left\{x_{n_{k}}\right\}$, weakly convergent to $\hat{x}:=E[\hat{\eta} X]$. Let us prove that $x_{n_{k}} \rightarrow \hat{x}$ (strongly) in $\mathbb{X}$; this would give the required compactness property. that

Assuming that $x_{n_{k}} \not \rightarrow \hat{x}$ in $\mathbb{X}$, we have a sequence $\left\{x_{k}^{*}\right\}$ of elements of the unit ball in $\mathbb{X}^{*}$ such

$$
\left\langle x_{n_{k}}-\hat{x}, x_{k}^{*}\right\rangle \not \supset 0 .
$$


By the Banach-Alaoglu theorem, we can assume without loss of generality that $x_{k}^{*} \rightarrow x^{*} *-$ weakly. Then

$$
\left\langle X, x_{k}^{*}\right\rangle \rightarrow\left\langle X, x^{*}\right\rangle,
$$

with probability 1 . By the uniform integrability of (4), this convergence also holds true in $L_{1}(\Omega, \sigma(X), P)$. Then

$$
\left\langle x_{n_{k}}, x_{k}^{*}\right\rangle=E \eta_{n_{k}}\left\langle X, x^{*}\right\rangle+E \eta_{n_{k}}\left(\left\langle X, x_{k}^{*}\right\rangle-\left\langle X, x^{*}\right\rangle\right) \rightarrow E \hat{\eta}\left\langle X, x^{*}\right\rangle=\left\langle\hat{x}, x^{*}\right\rangle
$$

because $\eta_{n_{k}} \rightarrow \hat{\eta}$ *-weakly in $L_{\infty}(\Omega, \sigma(X), P)$. The latter relation contradicts to (11). This proves compactness of $Z(\mu)$. For $\hat{Z}(\mu)$ and $D_{\alpha}(\mu), \alpha \in(0,1]$ the argument is completely analogous.

Statement (c). The proof here is completely analogous to the one of Theorem 3.5, [5] in the finite-dimensional setting, and hence we just sketch it. For $x^{*} \in X^{*}$, denote by $\mu_{x^{*}}$ respective one-dimensional projection of $\mu$; that is, the law of the random variable $\left\langle X, x^{*}\right\rangle$. The calculation from the proof of Theorem 3.4 in [5] shows that for arbitrary cylinder measures $\mu, \nu$ the inclusion $\hat{Z}(\mu) \subset \hat{Z}(\nu)$ yields the inclusion $\hat{Z}\left(\mu_{x^{*}}\right) \subset \hat{Z}\left(\nu_{x^{*}}\right), x^{*} \in \mathbb{X}^{*}$. Therefore, it follows from the identity $\hat{Z}(\mu)=\hat{Z}(\nu)$ that for every $x^{*} \in \mathbb{X}^{*}$ lift zonoids $\hat{Z}\left(\mu_{x^{*}}\right), \hat{Z}\left(\nu_{x^{*}}\right)$ coincide and hence (see Remark 3.2 in [5]) $\mu_{x^{*}}=\nu_{x^{*}}$. Because a cylinder measure is identified uniquely by its one-dimensional projections, we have then $\mu=\nu$.

Proposition 2. We have just proved that $Z(\mu)$ is a (non-empty) closed convex set. Then by by the second separability theorem (e.g. [9], Chapter II, Theorem 9.2)

$$
Z(\mu)=\bigcap_{H \in \mathcal{H}^{\mathbb{X}}: Z(\mu) \subset H} H
$$

Take $x^{*} \in \mathbb{S}_{\mathbb{X}^{*}}$ and consider half-spaces of the form $H=\left\{x:\left\langle x, x^{*}\right\rangle \leq a\right\}$. For every $\sigma(X)$ measurable $\eta: \Omega \rightarrow[0,1]$, one has

$$
E \eta\left\langle X, x^{*}\right\rangle \leq E\left\langle X, x^{*}\right\rangle_{+}=E h\left([0, X], x^{*}\right),
$$

which means that $Z(\mu) \subset H$ for any half-space of the above form with $a \geq E h\left([0, X], x^{*}\right)$. On the other hand, for $\eta=\mathbb{I}_{\left\langle X, x^{*}\right\rangle \geq 0}$ inequality (12) turns into the equality, which means that $Z(\mu) \not \subset H$ for any half-space of the above form with $a<E h\left([0, X], x^{*}\right)$. This proves statement 1. Statement 2 follows from statement 1.

3.2. Properties of zonoid trimmed regions. Here we prove statements $(\mathrm{d})-(\mathrm{h})$ of Theorem 1. Statement (d) follows directly from statement (a), because $D_{\alpha}(\mu)$ is the projection on $\mathbb{X}$ of the section of $\hat{Z}(\mu)$ by the affine hyper-plane $\{(t, x): t=\alpha\} \subset \mathbb{R} \times \mathbb{X}$. Statements (e) and (f) follow directly from the definition.

Statement $(g)$. By the statement (f), to prove the first part of the statement it is sufficient to show that

$$
D_{\alpha_{n}}(\mu) \rightarrow D_{\alpha}(\mu), \quad \alpha_{n} \uparrow \alpha<1
$$

in the Hausdorff distance. By the statement (e) we have $D_{\alpha}(\mu) \subset D_{\alpha_{n}}(\mu)$, and the only thing we need to prove is that for arbitrary sequence $x_{n} \in D_{\alpha_{n}}(\mu), n \geq 1$

$$
\operatorname{dist}\left(x_{n}, D_{\alpha}(\mu)\right) \rightarrow 0 .
$$

Let

$$
x_{n}=\frac{1}{\alpha_{n}} E\left[\eta_{n} X\right], \quad n \geq 1,
$$

where every $\eta_{n}: \Omega \rightarrow[0,1]$ is a $\sigma(X)$-measurable random variable such that $E \eta_{n}=\alpha_{n}$. We put

$$
\zeta_{n}=\eta_{n}+\frac{\alpha-\alpha_{n}}{1-\alpha_{n}}\left(1-\eta_{n}\right),
$$

then $\zeta_{n}$ is a $\sigma(X)$-measurable random variable taking values in $[0,1]$. Clearly, $E \zeta_{n}=\alpha$, and therefore $x_{n}^{\prime}:=(1 / \alpha) E\left[\zeta_{n} X\right]$ belongs to $D_{\alpha}(\mu)$. Because $\mu$ is centered, we have

$$
x_{n}^{\prime}=x_{n}-\frac{\alpha-\alpha_{n}}{1-\alpha_{n}} x_{n} .
$$


Not that every $x_{n}$ belongs to the set $D_{\alpha_{1}}(\mu)$, which is bounded by statement (d). Because $\alpha_{n} \rightarrow \alpha$ and $\alpha<1$, we have $\left\|x_{n}-x_{n}^{\prime}\right\| \rightarrow 0$, which proves (13).

The set $D_{1}(\mu)$ consists of one point $E[X]=0$. Hence, to prove the second part of the statement, we need to show that if $x=\left(1 / \alpha_{n}\right) E\left[\eta_{n} X\right]$ for some sequence of $\sigma(X)$-measurable random variables with $\alpha_{n}:=E \eta_{n} \rightarrow 1$, then $x=0$. Since $\mu$ is centered, $(-x)=\left(1 / \alpha_{n}\right) E[(1-$ $\left.\left.\eta_{n}\right) X\right]$. Clearly, $\left(1-\eta_{n}\right) \rightarrow 0$ in probability. Then by the Lebesque dominated convergence theorem we have

$$
E\left(1-\eta_{n}\right)\left\langle X, x^{*}\right\rangle \rightarrow 0, \quad x^{*} \in \mathbb{X}^{*} .
$$

This gives $\left\langle(-x), x^{*}\right\rangle=0, x^{*} \in \mathbb{X}^{*}$ and therefore $x=0$.

Statement $(h)$. First, let us show that for every $\alpha \in(0,1]$ the set $D_{\alpha}(\mu)$ is contained in every $H \in \mathcal{H}^{\mathbb{X}}$ with $\mu(H)=1$; this would yield $D_{0}(\mu) \subset \mathbf{S}(\mu)$. If $H=\mathbb{X}$, the inclusion is trivial. Otherwise $H=\left\{x:\left\langle x, x^{*}\right\rangle \geq a\right\}$ is an affine half-space, and we have $\left\langle X, x^{*}\right\rangle \geq a$ with probability 1 . Then for every random variable taking values in $[0,1]$ one has

$$
E\left[\eta\left\langle X, x^{*}\right\rangle\right] \geq a E \eta,
$$

which implies the required inclusion.

On the other hand, the closure of $D_{0}(\mu)$ is convex by statements (d), (e) and, of course, is closed. Then, by the second separability theorem, for any point $x$ which does not belong to this closure there exist $x^{*} \in \mathbb{X}^{*}, a \in \mathbb{R}$ such that

$$
a:=\inf _{y \in D_{0}(\mu)}\left\langle y, x^{*}\right\rangle>\left\langle x, x^{*}\right\rangle .
$$

It is easy to see that the affine half-space $H=\left\{x:\left\langle x, x^{*}\right\rangle \geq a\right\}$ satisfies $\mu(H)=1$. Indeed, if this is incorrect then $\mathbb{X} \backslash H=\left\{x:\left\langle x, x^{*}\right\rangle<a\right\}$ has non-zero measure $\mu$, and then the point

$$
z:=\frac{1}{\mu(\mathbb{X} \backslash H)} E\left[\mathbb{I}_{\mathbb{X} \backslash H}(X) X\right]
$$

belongs to $D_{0}(\mu)$. Since by the construction $\left\langle z, x^{*}\right\rangle<a$, this would contradict to the definition of $a$. Therefore, for arbitrary $x \notin H$ there exists some affine half-space $H$ with $\mu(H)=1$ such that $x \notin H$. This means that $x \notin \mathbf{S}(\mu)$, and consequently the closure of $D_{0}(\mu)$ coincides with whole $\mathbf{S}(\mu)$.

3.3. Barycentric representation. In this section, we use the properties $(\mathrm{d})$ - (h) of the family of zonoid $\alpha$-trimmed regions to prove Theorem 2 ,

First, we note that every $D_{\alpha}(\mu), \alpha \in(0,1)$ has a non-empty interior. Indeed, it follows from condition (ii) and the Baire category theorem that some $D_{\alpha}(\mu), \alpha \in(0,1)$ has a non-empty interior. Then the required statement follows from the property (f).

Next, let us proceed with the construction of the barycentric representation of the points $x \in D_{0}(\mu)$ in the form (22). Clearly, for $x=0$ there exists such representation with $H=\mathbb{X}$, and this representation is unique in the sense explained above. Consider arbitrary $x \in D_{0}(\mu)$, $x \neq 0$. There exists unique $\alpha=\alpha(x) \in(0,1)$ such that $x \in D_{\alpha}(\mu)$ and $x \notin D_{\beta}(\mu)$ for any $\beta>\alpha$; this follows from the property (e) in Theorem 1, By the continuity property (g), this yields $x \in \partial D_{\alpha}(\mu)$. Because $D_{\alpha}(\mu)$ has non-empty interior, we can apply the first separability theorem (e.g. 9], Chapter II, Theorem 9.1 and subsequent corollary), and get that there exists a support hyper-plane at the point $x$ to $D_{\alpha}(\mu)$. That is, there exists $x^{*} \in \mathbb{X}^{*} \backslash\{0\}$ such that

$$
\left\langle x, x^{*}\right\rangle \geq\left\langle y, x^{*}\right\rangle, \quad y \in D_{\alpha}(\mu) .
$$

By the condition (8), there exists $a$ such that

$$
\mu\left(\left\{x^{\prime}:\left\langle x^{\prime}, x^{*}\right\rangle \geq a\right\}\right)=\alpha .
$$

We put $f=\mathbf{I}_{A}(X), A=\left\{x^{\prime}:\left\langle x^{\prime}, x^{*}\right\rangle \geq a\right\}$. Then $f \in F_{\alpha}(\mu)$, and for every $g \in F_{\alpha}(\mu)$

$$
\begin{aligned}
E(f-g)\left\langle X, x^{*}\right\rangle & =E(f-g)\left(\left\langle X, x^{*}\right\rangle-a\right) \\
& =E\left(\left\langle X, x^{*}\right\rangle-a\right)\left((1-g) \mathbb{I}_{A}(X)\right)+E\left(\left\langle X, x^{*}\right\rangle-a\right)\left(-g \mathbb{I}_{\mathbb{X} \backslash A}(X)\right) \geq 0,
\end{aligned}
$$


because both expectations in the right hand side of (15) are non-negative. This means that

$$
\frac{1}{\alpha} E\left[\mathbf{I}_{A}(X) X\right]=B_{A}(\mu)
$$

is the unique point in the set $D_{\alpha}(\mu)$ which provides the extremum for the functional $y \mapsto\left\langle y, x^{*}\right\rangle$ at this set. Combined with (14), this gives the required representation (22) with $H=A$. The uniqueness of the representation follows from the strict inequality in (15), valid as soon as $f$ and $g$ do not coincide a.s.

Note that, obviously, $\mathbf{B}(\mu) \subset D_{0}(\mu)$. Because we have already proved the (unique) representation of any point of $D_{0}(\mu)$ as a barycenter of some set $H \in \mathcal{H}^{\mathbb{X}}$, this completes the proof of statement 2 .

Recall that $D_{0}(\mu)$ is convex and has a non-empty interior. Then (see [9], Chapter II, $\S 1.3$ ) the interior of $D_{0}(\mu)$ coincides with the interior of its closure. Combined with the property (h) from Theorem 1, this gives that every interior point of $\mathbf{S}(\mu)$ belongs to (the interior of) $D_{0}(\mu)$. On the other hand, since the interior of $\mathbf{S}(\mu)$ is non-empty, it follows from the first separability theorem that for every $x \in \mathbf{S}(\mu)$, which is not an interior point of $\mathbf{S}(\mu)$, there exist $x^{*} \in \mathbb{X} \backslash\{0\}$ and $a$ such that $\left\langle x, x^{*}\right\rangle=a$ and $\mu\left(x^{\prime}:\left\langle x^{\prime}, x^{*}\right\rangle \leq a\right)=1$. Then, using (8), it is easy to show that for every every point $y \in D_{0}(\mu)$ one has $\left\langle y, x^{*}\right\rangle<a$, and consequently $x \notin D_{0}(\mu)$. This means that $D_{0}(\mu)$ coincides with the interior of $\mathbf{S}(\mu)$, and completes the proof of statement 1.

\section{BARYCENTRIC COORDINATES}

We have mentioned briefly in the Introduction that the barycentric representation, established in Theorem 2, in some particularly interesting cases, makes it possible to introduce the barycentric coordinates on the initial space. Here we discuss this topic in details.

Assume that $\mu$ is a cylinder probability measure of type 1 on $\mathbb{X}$ which is centered and satisfies (8). Assume also that

$$
\mu(H \Delta G)>0 \quad \text { for every } H, G \in \mathcal{H}^{\mathbb{X}}, \quad H \neq G .
$$

Note that the above assumptions yield that every non-empty set $H \in \mathcal{H}^{\mathbb{X}}$ has positive measure $\mu$, and therefore the set $\mathbf{S}(\mu)$ coincides with whole $\mathbb{X}$.

For $x \in \mathbb{X}$ define

$$
\alpha(x)=\sup \left\{\alpha \in(0,1]: x \in D_{\alpha}(\mu)\right\},
$$

the zonoid data depth of the point $x$ w.r.t. $\mu$, see Definition 7.1 in [4]. For one exceptional point $x=0$, we have $\alpha(x)=1$. For all others, we have $\alpha(x) \in(0,1)$, and according to (16) and the proof from Section 3.3 there exist unique $x^{*} \in \mathbb{S}_{\mathbb{X}^{*}}$ and $a \in \mathbb{R}$ such that $x$ equals the barycenter of the half-space $H=\left\{x^{\prime}:\left\langle x^{\prime}, x^{*}\right\rangle \geq a\right\}$. Clearly, the pair $\left(a, x^{*}\right)$ determines $x$ uniquely, hence we can identify any point $x \in \mathbb{X} \backslash\{0\}$ by a pair

$$
\left(a, x^{*}\right) \in \mathbb{R} \times \mathbb{S}_{\mathbb{X}^{*}} .
$$

On the other hand, for $x \neq 0$ consider respective $x^{*} \in \mathbb{S}_{\mathbb{X}^{*}}$ and the value of the support function of $D_{\alpha(x)}(\mu)$ on this $x^{*}$ :

$$
h=h\left(D_{\alpha(x)}(\mu), x^{*}\right) .
$$

It can be seen easily that $x$ is the unique point of $D_{\alpha(x)}(\mu)$ on the affine hyper-plane $\left\{x^{\prime}\right.$ : $\left.\left\langle x^{\prime}, x^{*}\right\rangle=h\right\}$. Hence, there is another possibility to identify a point $x \in \mathbb{X} \backslash\{0\}$ by a pair

$$
\left(h, x^{*}\right) \in(0, \infty) \times \mathbb{S}_{\mathbb{X}^{*}} .
$$

Finally, given a set $D_{\alpha}(\mu)$ and $x^{*} \in \mathbb{S}_{\mathbb{X}^{*}}$, one can uniquely define $h>0$ such that the affine hyper-plane $\left\{x^{\prime}:\left\langle x^{\prime}, x^{*}\right\rangle=h\right\}$ is a support hyper-plane for $D_{\alpha}(\mu)$. Hence, we can also identify a point $x \in \mathbb{X} \backslash\{0\}$ by a pair

$$
\left(\alpha, x^{*}\right) \in(0,1) \times \mathbb{S}_{\mathbb{X}^{*}},
$$

where the first coordinate coincides with the zonoid data depth $\alpha(x)$. 
Let us summarise. We have represented $\mathbb{X}$ as a disjunctive union

$$
\bigsqcup_{\alpha \in(0,1]} Z_{\alpha}(\mu)
$$

where $Z_{\alpha}(\mu)$ denotes the set of points with the zonoid data depth w.r.t. $\mu$ equal $\alpha$. The set $Z_{1}(\mu)$ consists of one point 0 . For $\alpha \in(0,1)$, any point $x \in Z_{\alpha}(\mu)$ is identified by respective $x^{*} \in \mathbb{S}_{\mathbb{X}^{*}}$, which can be naturally understood as the unique "outside tangent direction" for $Z_{\alpha}(\mu)$ at the point $x$. This leads to the representation (19). Without loss of the uniqueness of representation property, the scalar $\alpha$ can be replaced either by $h=\left\langle x, x^{*}\right\rangle$ (which would lead to (18)), or by $a$ such that $x$ is the barycenter of the affine half-space $H=\left\{x^{\prime}:\left\langle x^{\prime}, x^{*}\right\rangle \geq a\right\}$ (this would lead to (17)).

\section{Barycentric Representation on a space With a $\sigma$-ADditive measure}

Let $\mathbb{X}$ be a separable (not necessarily reflexive) Banach space and $\mu$ be a probability measure on the Borel $\sigma$-algebra in $\mathbb{X}$. Assume that $\mu$ has a weak order of some order $p>1$; that is, (6) holds true. Then Theorem 2 is not applicable because its condition (ii) fails. Here we discuss the structure of the zonoid span $\mathcal{L}(\mu)$, and give a version of the barycentric representation valid in that case. Without loss of generality we assume $\mu$ to be centered.

To study the properties of the zonoid $\operatorname{span} \mathcal{L}(\mu)$, it is convenient to use the objects related to the notion of a measurable linear functional; e.g. 3. Let us recall briefly respective constructions.

For $r \in[1, p]$, denote the canonical embedding of $\mathbb{X}^{*}$ into $L_{r}(\mathbb{X}, \mu)$ by $T_{r, \mu}$. Define the space $\mathbb{H}_{r, \mu}(\mathbb{X})$ as the closure of the range of $T_{r, \mu} ;$ any element in $\mathbb{H}_{r, \mu}(\mathbb{X})$ is called an ( $L_{r}$-integrable) measurable linear functional.

For any $g \in L_{\infty}(\mathbb{X}, \mu)$ the integral

$$
\int_{\mathbb{X}} g(x) x \mu(d x) \in \mathbb{X}
$$

is well defined, see [10], Chapter II, $\S 3$. It can be verified easily that the adjoint operator $T_{1, \mu}^{*}: L_{\infty}(\mathbb{X}, \mu) \rightarrow \mathbb{X}^{* *}$ has the form

$$
T_{1, \mu}^{*} g=\int_{\mathbb{X}} g(x) x \mu(d x), \quad g \in L_{\infty}(\mathbb{X}, \mu),
$$

and in particular takes its values in $\mathbb{X} \subset \mathbb{X}^{* *}$.

Proposition 3. (1) The set $\mathcal{L}(\mu)$ equals the range of $T_{1, \mu}^{*}$.

(2) The norm $\|\cdot\|_{\mathcal{L}}$ is equivalent to the norm $\|\cdot\|_{\mathcal{R}}$ defined by

$$
\|x\|_{\mathcal{R}}=\inf _{g \in L_{\infty}(\mathbb{X}, \mu): T_{1, \mu}^{*} g=x}\|g\|_{L_{\infty}(\mathbb{X}, \mu)}, \quad x \in \mathcal{L}(\mu)=\mathcal{R}\left(T_{1, \mu}^{*}\right) .
$$

The proof follows directly from the definitions of the respective objects, and hence we omit the details.

The statement of Proposition 3 can be made even more precise. Consider the dual space $\left[\mathbb{H}_{1}(\mathbb{X}, \mu)\right]^{*}$; that is, the factor-space in $L_{\infty}(\mathbb{X}, \mu)$ w.r.t. the equivalence

$$
g_{1} \sim g_{2} \Leftrightarrow \int_{\mathbb{X}}\left(g_{1}(x)-g_{2}(x)\right)\left\langle x, x^{*}\right\rangle \mu(d x), \quad x^{*} \in \mathbb{X}^{*},
$$

see [8], Chapter 4. One can see easily that for any $g \in L_{\infty}(\mathbb{X}, \mu)$

$$
T_{1, \mu}^{*} g=0 \Leftrightarrow g \sim 0 .
$$

This means that $T_{1, \mu}^{*}$ can be naturally understood as a linear operator $\left[\mathbb{H}_{1}(\mathbb{X}, \mu)\right]^{*} \rightarrow \mathcal{L}(\mu)$. In addition, the norm in the space $\left[\mathbb{H}_{1}(\mathbb{X}, \mu)\right]^{*}$ is defined by the relation

$$
\|g\|_{\left[\mathbb{H}_{1}(\mathbb{X}, \mu)\right]^{*}}=\inf _{\tilde{g} \sim g}\|\tilde{g}\|_{L_{\infty}(\mathbb{X}, \mu)} .
$$

Comparing this with the expression in the right hand of (20), we obtain the following. 
Corollary 1. The zonoid span $\mathcal{L}(\mu)$ is a Banach space isomorphic to the dual space of the space of $\mathbb{H}_{1}(\mathbb{X}, \mu)$ of $L_{1}$-integrable measurable linear functionals. Respective isomorphism is provided by the operator $T_{1, \mu}^{*}$.

Now, we are ready to give a version of the barycentric representation valid in the case under the consideration. Denote by $\mathcal{H}_{1, \mu}^{\mathbb{X}}$ the family of the sets of the form

$$
\{x: h(x) \geq a\}, \quad h \in \mathbb{H}_{1, \mu}(\mathbb{X}), \quad a \in \mathbb{R} .
$$

Theorem 3. Assume that

(a) for every $h \in \mathbb{H}_{1, \mu}(\mathbb{X}) \backslash\{0\}, a \in \mathbb{R}$

$$
\mu(\{x: h(x)=a\})=0 ;
$$

(b) for some $r>1, \mathbb{H}_{1, \mu}(\mathbb{X})=\mathbb{H}_{r, \mu}(\mathbb{X})$.

Then the following holds true.

(1) The set $\mathbf{B}(\mu)=\left\{B_{A}(\mu), \mu(A)>0\right\}$ of all barycenters w.r.t. $\mu$ equals the interior of $\mathbf{S}(\mu) \cap \mathcal{L}(\mu)$ w.r.t. to the topology induced by the norm $\|\cdot\|_{\mathcal{L}}$.

(2) Any point $x \in \mathbf{B}(\mu)$ has a representation in the form

$$
x=B_{H}(\mu), \quad H \in \mathcal{H}_{1, \mu}^{\mathbb{X}}, \quad \mu(H)>0 .
$$

This representation is unique in the following sense: if $B_{H}(\mu)=B_{G}(\mu)$ for some $H, G \in$ $\mathcal{H}_{1, \mu}^{\mathbb{X}}$, then

$$
\mu(H \Delta G)=0 .
$$

Proof. The identity $\mathbb{H}_{1, \mu}(\mathbb{X})=\mathbb{H}_{r, \mu}(\mathbb{X})$ yields that the Banach spaces $\left[\mathbb{H}_{1, \mu}(\mathbb{X})\right]^{*}$ and $\left[\mathbb{H}_{r, \mu}(\mathbb{X})\right]^{*}$ are isometric. Denote $r^{\prime}=r /(r-1)$, then $\left[\mathbb{H}_{r, \mu}(\mathbb{X})\right]^{*}$ is a factor-space in $L_{r^{\prime}}(\mathbb{X}, \mu)$ w.r.t. to the equivalence (21). Using the fact that $L_{r^{\prime}}(\mathbb{X}, \mu)$ is reflexive, it is easy to show that every space $\left[\mathbb{H}_{r, \mu}(\mathbb{X})\right]^{*},\left[\mathbb{H}_{1, \mu}(\mathbb{X})\right]^{*}$, and $\mathcal{L}(\mu)$ is reflexive, as well.

Denote $\tilde{\mathbb{X}}=\mathcal{L}(\mu)$, then $\widetilde{\mathbb{X}}^{*}$ is isometric to $\mathbb{H}_{1, \mu}(\mathbb{X})$. Denote by $J: \mathbb{H}_{1, \mu}(\mathbb{X}) \rightarrow \tilde{\mathbb{X}}^{*}$ respective isometry, and define on the probability space $(\Omega, \mathcal{F}, P)=(\mathbb{X}, \mathcal{B}(\mathbb{X}), \mu)$ a generalised random element $\tilde{X}$ valued in $\tilde{\mathbb{X}}$ by

$$
\langle\tilde{X}, J h\rangle=h, \quad h \in \mathbb{H}_{1, \mu}(\mathbb{X}) .
$$

By the construction, the law $\tilde{\mu}$ of $\tilde{X}$ is a cylinder measure of the type 1 . In addition, $\sigma(\tilde{X})$ coincides with $\mathcal{F}$, which implies that the zonoid, the lift zonoid, and the zonoid $\alpha$-trimmed regions for $\mu$ are equal to the images of the same objects for $\tilde{\mu}$ under the natural embedding $\mathcal{L}(\mu) \rightarrow \mathbb{X}$. Therefore the required statements follows from Theorem 2 applied to $\tilde{\mu}$.

Remark 5. Condition (b) in the above proof is used to guarantee that $\mathcal{L}(\mu)$ is a reflexive Banach space. This condition holds true in particularly interesting cases, i.e. for a Gaussian measure $\mu$ or, more generally, for a convex measure $\mu$; see 1 . However, this is a non-trivial structural assumption on $\mu$, and one can be interested in other conditions which would provide $\mathcal{L}(\mu)$ to be reflexive. This is a subject for a further investigation.

\section{EXAMPLE}

We illustrate the above results by an example of a Gaussian measure $\mu$, where the calculations are most simple. The results below are not genuinely new; e.g. statement 1 in Proposition 5 is already known, see Theorem 4.3 in [2].

Let $\mathbb{X}$ be a separable Hilbert space, and $\mu$ be a centered cylinder Gaussian measure on $\mathbb{X}$ with the identity covariance operator. By the definition, this means that for the respective generalized random element $X$

$$
(X, x)_{\mathbb{X}} \sim \mathcal{N}\left(0,\|x\|_{\mathbb{X}}^{2}\right), \quad x \in \mathbb{X}
$$

here we identify $\mathbb{X}^{*}$ with $\mathbb{X}$ and write $(\cdot, \cdot)_{\mathbb{X}}$ instead of $\langle\cdot, \cdot\rangle$. Clearly, $\mu$ satisfies (8) and (16).

The law of $X$ is rotationally invariant; that is, $U X$ has the same distribution with $X$ for any unitary operator $U: \mathbb{X} \rightarrow \mathbb{X}$. Then it is easy to see that every $D_{\alpha}(\mu)$ is a closed ball in $\mathbb{X}$; 
denote the radius of this ball by $r(\alpha)$. We can determine this radius easily, using the arguments from Section 4. Namely, for every point $x$ on the boundary of $D_{\alpha}(\mu)$ respective "outside tangent direction" equals $\|x\|_{\mathbb{X}}^{-1} x$. Then $x$ can be uniquely represented as the barycenter of the halfspace

$$
H_{x}=\left\{x^{\prime}:\left(x^{\prime},\|x\|_{\mathbb{X}}^{-1} x\right)_{\mathbb{X}} \geq a(x)\right\},
$$

where the scalar $a=a(x)$ is determined by the relation $\mu\left(H_{x}\right)=\alpha$. Because

$$
\mu\left(H_{x}\right)=P\left(\left(X,\|x\|_{\mathbb{X}}^{-1} x\right)_{\mathbb{X}} \geq a(x)\right),
$$

and $\left(X,\|x\|_{\mathbb{X}}^{-1} x\right)$ has a standard Gaussian distribution, we have

$$
a(x)=\Phi^{-1}(1-\alpha), \quad \Phi(u):=\int_{-\infty}^{u} \varphi(v) d v, \quad \varphi(u):=\frac{1}{\sqrt{2 \pi}} e^{-u^{2} / 2} .
$$

Then

$$
\begin{aligned}
r(\alpha) & =\|x\|_{\mathbb{X}}=\left(x,\|x\|_{\mathbb{X}}^{-1} x\right)=\frac{1}{\alpha} E\left(X,\|x\|_{\mathbb{X}}^{-1} x\right)_{\mathbb{X}} \mathbf{I}_{\left(X,\|x\|_{\mathbb{X}}^{-1} x\right)_{\mathbb{X}} \geq a(x)} \\
& =\frac{1}{\alpha \sqrt{2 \pi}} \int_{a(x)}^{\infty} u e^{-u^{2} / 2} d u=\frac{1}{\alpha} I(\alpha),
\end{aligned}
$$

where $I:=\varphi \circ \Phi^{-1}$ is the so-called Gauss isoperimetric function; here we have used that $\Phi^{-1}(1-\alpha)=-\Phi^{-1}(\alpha)$ and that $\varphi$ is even. Remark that then for $x \neq 0$ respective zonoid data depth equals $r^{-1}\left(\|x\|_{\mathbb{X}}\right)$, and hence

$$
a(x)=-\Phi^{-1}\left(r^{-1}\left(\|x\|_{\mathbb{X}}\right)\right)=-G^{-1}\left(\|x\|_{\mathbb{X}}\right), \quad \text { where } \quad G(u):=\frac{\varphi(u)}{\Phi(u)} ;
$$

note that the function $G: \mathbb{R} \rightarrow(0, \infty)$ is monotonous. Therefore, we have proved the following.

Proposition 4. Let $\mu$ be a centered cylinder Gaussian measure on $\mathbb{X}$ with the identity covariance operator.

Then the following holds.

(1) Every zonoid $\alpha$-trimmed region $D_{\alpha}(\mu), \alpha \in(0,1)$ equals the ball with the radius $r(\alpha)=$ $\alpha^{-1} I(\alpha)$.

(2) Every point $x \neq 0$ has a unique representation as a barycenter w.r.t. $\mu$ of the half-space (23) with $a(x)=-G^{-1}\left(\|x\|_{\mathbb{X}}\right)$.

The case of $\mu$ being a centered $\sigma$-finite Gaussian measure on a separable Banach space $\mathbb{X}$, can be reduced to the above, like it was done in Section 5. Because convergence in probability of a Gaussian sequence yields $L_{p}$-convergence for every $p \geq 1$, we have that all spaces $\mathbb{H}_{p, \mu}(\mathbb{X}), p \geq 1$ now coincide. Respective space is called the reproducing kernel space for the Gaussian measure $\mu$; we denote it by $\mathbb{H}_{\mu}$. Because $\mathbb{H}_{\mu}=\mathbb{H}_{2, \mu}(\mathbb{X})$ can be considered as the Hilbert space, $\mathcal{L}(\mu)$ equals the image of $\mathbb{H}_{\mu}$ under the natural embedding $J_{\mu}: \mathbb{H}_{\mu} \rightarrow \mathbb{X}$ (this image is called usually the Cameron-Martin space of $\mu$ ). On this space, $\mu$ generates a centered Gaussian cylinder measure $\tilde{\mu}$ with the identity covariance operator. Applying Proposition 4 to $\tilde{\mu}$, we arrive at following.

Proposition 5. Let $\mu$ be a a centered $\sigma$-finite Gaussian measure on a separable Banach space $\mathbb{X}$.

Then the following holds.

(1) Every zonoid $\alpha$-trimmed region $D_{\alpha}(\mu), \alpha \in(0,1)$ equals the image of the ball in $\mathbb{H}_{\mu}$ with the radius $r(\alpha)=\alpha^{-1} I(\alpha)$ under the natural embedding of $\mathbb{H}_{\mu}$ into $\mathbb{X}$.

(2) Every point $x \neq 0$ in the Cameron-Martin space has a unique representation as a barycenter w.r.t. $\mu$ of the measurable half-space

$$
H_{x}=\left\{x^{\prime}:\left(x^{\prime},\left\|J_{\mu}^{-1} x\right\|_{\mathbb{H}_{\mu}}^{-1} J_{\mu}^{-1} x\right)_{\mathbb{H}_{\mu}} \geq a(x)\right\}, \quad a(x)=-G^{-1}\left(\left\|J_{\mu}^{-1} x\right\|_{\mathbb{H}_{\mu}}\right),
$$

where $(\cdot, h)_{\mathbb{H}_{\mu}}$ denotes the linear measurable functional respective to $h \in \mathbb{H}_{\mu}$. 
Acknowledgement. The authors a grateful to Ilya Molchanov, who have turned their attention to the lift zonoid concept.

\section{REFERENCES}

[1] C. Borell C. Convex measures on locally convex spaces. Ark. Math., 12, 239 - 252, 1974.

[2] C. Borell. Zonoids induced by Gauss measure with an application to risk aversion. Alea, 6, 133 - 147, 2009.

[3] A. A. Dorogovtsev. Measurable functionals and finitely absolutely continuous measures on Banach spaces. Ukrain. Mat. Zh. 52:9, 1194 - 1204, 2000. (Russian; translation in Ukrainian Math. Jour. 52:9, 1366 - 1379 , 2001).

[4] G. A. Koshevoy and K. Mosler. Zonoid trimming for multivaiate distributions. Ann. Stat., 25:5, 1998 - 2017 , 1997.

[5] G. A. Koshevoy and K. Mosler. Lift zonoids, random convex hulls and the variability of random vectors. Bernoulli, 4, 377 - 399, 1998.

[6] I. Molchanov and M. Schmutz. Exchangeability type properties of asset prices. Adv. Appl. Probab., 43, 666 $-687,2011$.

[7] I. Molchanov, M. Schmutz, and K. Stucki. Invariance properties of random vectors and stochastic processes based on the zonoid concept. arXiv:1203.6085

[8] W. Rudin. Functional Analysis. McGraw-Hill, New-York, 1973.

[9] H. H. Schaefer. Topological vector spaces. New York: Springer-Verlag, 1971.

[10] N. Vakhania, V. Tarieladze, and S. Chobanyan. Probability distributions on Banach spaces. New York: Springer-Verlag, 1987.

Institute of Mathematics Nat. Acad. Sci. of Ukraine, Tereshchenkivska str. 3, 01601, Kyiv, UKRAINE

E-mail address: kulik@imath.kiev.ua

National Taras Shevchenko University of Kyiv, Academician Glushkov pr. 4-e, 03127, Kyiv, UKRAINE.

E-mail address: tymoschkevych@gmail.com 